\section{Reward-related limbic memory and stimulation of the cannabinoid system: An upgrade in value attribution?}

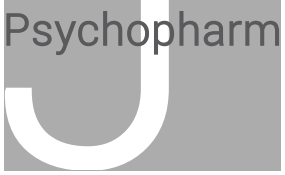

Journal of Psychopharmacology 1-11

(c) The Author(s) 2017

Reprints and permissions:

sagepub.co.uk/journalsPermissions.nav DOI: $10.1177 / 0269881117725683$ journals.sagepub.com/home/jop

\author{
Anna Brancato, Angela Cavallaro, Gianluca Lavanco, \\ Fulvio Plescia and Carla Cannizzaro
}

\begin{abstract}
While a lot is known about the mechanisms promoting aversive learning, the impact of rewarding factors on memory has received comparatively less attention. This research investigates reward-related explicit memory in male rats, by taking advantage of the emotional-object recognition test. This is based on the prior association, during conditioned learning, between a rewarding experience (the encounter with a receptive female rat) and an object; afterwards rat discrimination and recognition of the 'emotional object' is recorded in the presence of a novel object, as a measure of positive limbic memory formation. Since endocannabinoids are critical for processing reward and motivation, the consequences of the stimulation of cannabinoid signalling are also assessed by the administration of WIN 55,212-2 at pre- and post-conditioning time.

Our results show that rats encode the association between object and rewarding experience, form positive limbic memory of the emotional object, and retrieve this information in the face of novelty. Stimulation of the cannabinoid system at pre-conditioning time is able to strengthen reward-related explicit memory in the presence of novelty, whereas post-conditioning activation increases approach behaviour to novel stimuli.

The assessment of limbic memory by the emotional-object recognition test can help unveiling the addictive and confounding properties of psychotropic drugs.
\end{abstract}

\title{
Keywords
}

Memory, reward-conditioning, emotional-object recognition, cannabinoid stimulation

\section{Introduction}

Research on episodic memory, that is remembering what happened, as well as where and when it happened, has been traditionally exploring memory systems that receive and store information about neutral episodes or events and their spatial and temporal contexts (Brown et al., 2010; Cannizzaro et al., 2005, 2007; Chao et al., 2017). However, emotional events hold a privileged status in memory. The enhancement of consciously accessible memory for emotional stimuli, here called limbic memory, is an important prerequisite for successful goal-oriented behaviours (Christianson and Engelberg, 1997; LeDoux, 1992; Niendam et al., 2012; Ochsner and Gross, 2005; Ramirez et al., 2015; Tonegawa et al., 2015).

Notwithstanding, in preclinical research the majority of studies into associative learning and memory have focused on spatial negative states, particularly fear, anxiety, and 'stress' (Lang et al., 2000; Shin and Liberzon, 2010), relying on simple or variations of the classical Pavlovian conditioning procedure (Brancato et al., 2016a; LeDoux, 2000). Comparatively, the impact of rewarding factors on memory processes has received less attention. Besides, consumption of reward (e.g. palatable food, mating, cocaine), or seeking for novelty produce hedonic consequences, that might initiate learning processes, consolidate value of emotional cues and promote associative emotional memory (Asensio et al., 2010; Harb et al., 2014; Kelley et al., 2005; Murphy et al., 2012; Peretto and Paredes, 2014; Wang et al., 2014; Wells et al., 2011).
Among the experimental approaches that have been used to study memory for natural and drug-related reward in laboratory animals, conditioning place preference (CPP) is one of the most popular (Bardo and Bevins, 2000; Trezza et al., 2011; Tzschentke, 1998, 2007). The acquisition of preference for the compartment where the rewarding experience (sex intercourse, drug or food intake) took place has been interpreted as reward or drug-related memory. (Ågmo and Berenfeld, 1990; Harding and McGinnis, 2004; Hughes et al., 1990; Kippin and Van der Kooy, 2003; Mehrara and Baum, 1990; Miller and Baum, 1987).

As a matter of fact, place conditioning is a relevant measure of reward such as intracranial self-stimulation, progressive ratio responding, sucrose intake, etc. (Cunningham and Zerizef, 2014; Forbes et al., 1996), that focuses on automatic or implicit rewardevoked responses, rather than on the formation of declarative memory of rewarding experiences (Paredes, 2009). This complex

Department of Sciences for Health Promotion and Mother and Child Care 'G. D'Alessandro', University of Palermo, Palermo, Italy

\section{Corresponding author:}

Carla Cannizzaro, University of Palermo, Department of Sciences for Health Promotion and Mother and Child Care 'G. D'Alessandro', Via del Vespro 129, 90127 Palermo, Italy.

Email: carla.cannizzaro@unipa.it 
function is, instead, better explored by the object-female association task as developed by Ramirez et al. (2015). More generally object conditioning with aversive or appetitive stimuli such as footshock, food, novel objects, or socio-sexual behaviour, could be used in addition to CPP because they provide an insight into explicit memory of emotional experiences (Brancato et al., 2016a). Notably, reward modulates attention and memory formation, to a degree dependent on individual emotional traits (Cohen and Ranganath, 2005; Hahn et al., 2009).

In this regard, the interplay between the individual emotional milieu and the integration of value attribution and memory formation can be deeply affected by different psychotropic compounds (Cacace et al., 2011; Plescia et al., 2015) that produce aberrant behavioural responses to the environment (Brancato et al., 2014, 2016b; Cacace et al., 2012; Cannizzaro et al., 2010; Volkow et al., 2012). With relevance to this subject, research from both clinical and basic neuroscience shows that the brain's endocannabinoid system plays a well-established role in the processing of aversive emotional memory. Moreover, considerable evidence links the cannabinoid system to the regulation of reward-related processing (Loureiro et al., 2015, 2016): specifically, CB1 receptor activation enhances CPP after methamphetamine-, nicotine- and morphineconditioning and is also essential for extinction of conditioned fear associations (De Carvalho et al., 2014; Fang et al., 2011; Karimi et al., 2013; Marsicano et al., 2002; Yu et al., 2009; Zhang et al., 2011).

Given these premises, the first aim of this research was to explore the formation of reward-related limbic memory, by taking advantage of the emotional-object recognition (EOR) test. This procedure is based on the association between an emotionally salient experience (i.e. the encounter with a receptive female rat) and an object, in order to assess rat's ability to discriminate the 'emotional object', on the basis of the formation of positive limbic memory.

The second goal consisted in the evaluation of the activation of cannabinoid system on the encoding and retrieval of positive limbic memory, by the administration of the cannabinoid receptor agonist WIN 55,212-2 (WIN). The specific involvement of the CB1 cannabinoid receptor was assessed by the pharmacological pre-treatment with the CB1 receptor antagonist AM281 (Plescia et al., 2013, 2014a), before WIN administration.

This research would contribute to assess whether specific drugs, or neuropsychological disturbances, might interfere with the formation of positive limbic memory traces, thus jeopardizing value attribution and adaptive behavioural responses.

\section{Materials and methods}

\section{Animals}

Male Wistar rats (Harlan, Udine, Italy; $n=78$; weight: $250 \pm$ $20 \mathrm{gm})$, which were experimentally and sexually naïve, were housed in pairs in standard rat cages $(40 \mathrm{~cm} \times 60 \mathrm{~cm} \times 20 \mathrm{~cm}$ in height), with ad libitum access to water and food (Mucedola, Italy). The colony room was maintained under controlled environmental conditions (temperature $22 \pm 2{ }^{\circ} \mathrm{C}$; humidity $55 \pm 5 \%$ ) on a 12-h light/dark cycle. Animals were gently handled for 3 min per day for a week before the experimental sessions.

Rats were individually housed, in order to acclimatise to their single home cages for 3 days, before they would encounter a receptive female rat, which was selected by visual identification of the vaginal opening in pro-oestrus or oestrus (Byers et al., 2012).
On the test day, animals were brought into the laboratory 60 min before the experimental sessions. The experiments were carried out in sound-isolated rooms between 9:00 and 17:00. Animal performance was recorded on videotape and monitored in an adjacent room. All the experiments were conducted in accordance with the regulations of the Committee for the Protection and Use of Animals of the University of Palermo, Italy, in accordance with current Italian legislation on animal experimentation (D.L. 116/92) and the European Directive (2010/63/EU) on the care and use of laboratory animals. All efforts were made to minimise the number of animals used, and possible distress.

\section{Drugs}

The cannabinoid agonist WIN 55, 212-2 mesylate (WIN; Tocris Bioscience, UK) and the CB1 receptor antagonist AM281 (Sigma, Italy) were initially dissolved in 3\% ethanol; followed by $3 \%$ Tween 20 and 94\% saline (Cannizzaro et al., 2016). WIN was administered intraperitoneally (i.p.) at a dose of $1 \mathrm{mg} / \mathrm{kg}$ (volume of $1 \mathrm{~mL} / \mathrm{kg}$ ), $30 \mathrm{~min}$ before the cued reward-conditioned learning session (pre-conditioning WIN) or 30 min before the EOR experiment (post-conditioning WIN). WIN dosage was chosen on the basis of previous studies (Brancato et al., 2016; Darmani, 2001; Järbe, 2006), in order to avoid unspecific effects due to neural and locomotor impairment (Rizzo et al., 2014). AM281 was administered i.p. at a dose of $2 \mathrm{mg} / \mathrm{kg}, 30 \mathrm{~min}$ before WIN (Suenaga and Ichitani, 2008). Control rats received the same volume of vehicle at the same time points $(1 \mathrm{~mL} / \mathrm{kg})$.

\section{Emotional-object recognition (EOR) test}

Positive limbic memory was explored by using a cued rewardconditioned learning task, here named the EOR test (modified from Ramirez et al., 2015). It is composed of two following sessions: cued reward-conditioned learning and emotional-object recognition. The procedure is schematically summarised in Figure 1.

The EOR test employed two distinct contexts placed in different rooms. The Context A chamber was a rectangular arena with a white floor, $100 \mathrm{~cm}$ long, $30 \mathrm{~cm}$ wide and $43 \mathrm{~cm}$ high. A left and a right zone $(40 \times 30 \mathrm{~cm})$ on both ends of the Context A chamber, as well as a neutral zone in the centre of the box $(20 \times 30 \mathrm{~cm})$ represented the arena setting. The Context B chamber was the rat's home cage.

All rats were allowed into the Context B chamber for object habituation and cued reward-conditioned learning; the Context A chamber was employed for the EOR experiment. Rat behaviour was recorded and analysed by ANY MAZE Video Tracking System (Ugo Basile, Italy).

Two nontoxic hard-plastic objects, a pepper $(3 \times 3 \times 4 \mathrm{~cm})$ and a ball ( $3.5 \mathrm{~cm}$ diameter), were used and counterbalanced: the target object/emotional object appeared on both cued reward-conditioned learning and during EOR; the novel object was introduced during the EOR experiment, together with the emotional object.

\section{Experimental procedure}

Cued reward-conditioned learning. All rats were carried in the middle of a well-lit room into their home cages (Context B chamber). The target object was introduced into the cages and rats explored it for $3 \mathrm{~min}$. Afterwards a receptive female rat, was introduced in the Context B chamber and remained there for 27 


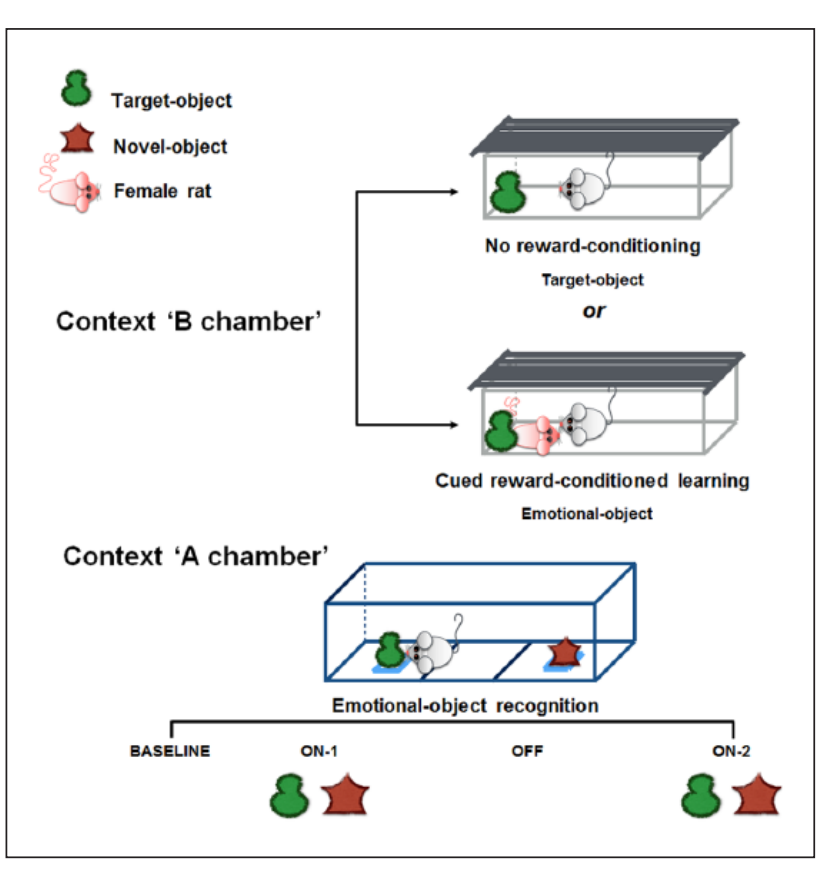

Figure 1. Schematic summary of the emotional-object recognition test.

min in the presence of the target object that is consequently named 'emotional object'. The use of the animal home cage assured a comfortable environment for reward-conditioning.

30 min after the beginning of the session, the emotional object and female rat were removed and male rats returned to their holding room for a retention interval of $4 \mathrm{~h}$.

\section{Emotional-object recognition}

At $4 \mathrm{~h}$ after the cued reward-conditioned learning, animals ( $\mathrm{R}$ group) were put in the neutral zone of the empty Context A chamber and allowed to explore freely for $5 \mathrm{~min}$. The tracking software monitored the amount of time that they spent in each compartment (baseline; BSL). Each rat's least preferred compartment was assigned to be the emotional-object paired compartment. Afterwards, the Context A chamber was customised with two nontoxic objects that were placed against the end walls of the left and the right zones of the arena: the least favourite zone, or target zone, hosted the emotional object experienced during cued reward-conditioned learning; a novel object was placed in the favourite zone of the chamber. This strategy helps the experimenter to nullify the interference of spatial preference with the exploration of the emotional object.

Both objects were thoroughly cleaned to prevent olfactory cues. Rats were allowed to explore the objects from min 5 to 8 (epoch ON-1) and from min 11 to 14 (epoch ON-2). During min 8 to 11, the objects were removed from the arena (epoch OFF). Finally, rats were placed in their home cages and sent back to the holding room. At the end of each experimental session both the objects and the arena were cleaned with a $70 \%$ solution of isopropanol. Time spent on exploring objects and zones was recorded along the epochs using the ANY MAZE Video Tracking System (Ugo Basile, Italy). Object exploration time was quantified as duration of rat approach to the objects within a $2 \mathrm{~cm}^{2}$ area around the objects (Plescia et al., 2014b).
The target-zone preference rate referred to the increase in the time spent in the target zone hosting the emotional object, over the time spent on both left and right zones, expressed as a percentage, with respect to the BSL. The difference score ON-BSL was measured as the difference between the total time in the target zone during $\mathrm{ON}(\mathrm{ON}-1+\mathrm{ON}-2)$ and time in the target zone during BSL. The EOR index (time spent on emotional object / time spent on novel + emotional- object) was calculated and expressed as a normalised value to the average reference values of respective control groups.

Experiment 1. EOR test - validation. In order to establish whether the acquisition of reward-associated memory traces occurred in the R group, another batch of male rats did not experience the female encounter and was only exposed to the 'target object' during the conditioned learning experiment (no reward; NoR group). In the EOR experiment, the NoR group's preference for the familiar target object and the target zone was recorded in the presence of a novel object.

Target-object exploration time, target-object recognition index, target-zone preference rate and difference score ON-BSL were compared with the R group's approaching behaviour to the emotional object in the EOR experiment ( $n=10$ per group).

Moreover, in order to control the effect of female receptivity, a third batch of male rats $(n=10)$ was exposed to nonreceptive female rats, which were not in pro-oestrous or oestrous stage (Byers et al., 2012) during the cued reward-conditioned learning. Their behavioural pattern in the cued reward-conditioned learning and in the EOR test was compared with the $\mathrm{R}$ group's.

Experiment 2. Effects of WIN on positive limbic memory in the EOR test. In order to assess the consequences of cannabinoid system stimulation on acquisition or retrieval of rewardassociated memory traces in the EOR test, male rats were administered with WIN (1 mg/kg i.p.) or vehicle (VEH) $30 \mathrm{~min}$ before the cued reward-conditioned learning (pre-conditioning time), or $30 \mathrm{~min}$ before the EOR experiment (post-conditioning time) ( $n=8$ per group). Target-object exploration time, targetobject recognition index, target-zone preference rate and difference score ON-BSL were assessed in Context A chamber.

The specificity of WIN effect on positive limbic memory was also tested through the pharmacological inhibition of CB1 signalling 30 min before WIN administration, by means of AM281 pretreatment $(2 \mathrm{mg} / \mathrm{kg}$, i.p. $)$.

The occurrence of nonspecific effects of pre-conditioning WIN during the cued reward-conditioned learning was assessed by measurement of the duration of anogenital sniffing towards the female rat and object exploration during the cued rewardconditioned learning experiment from the video records, as measures of socio-sexual behaviour (Chu and Ågmo, 2015) and object exploratory behaviour, respectively.

Experiment 3. Behavioural reactivity assessment. Potential motor impairment, which might be due to the encounter with the female rat or to WIN administration (at pre- and post-conditioning time), was assessed during 5 min-epoch BSL by ANY MAZE (Ugo Basile, Italy). The apparatus, which produces a quali-quantitative mapping of the locomotor pattern, measured total distance travelled $(\mathrm{m})$. 


\section{Statistical analysis}

All data were expressed as mean \pm SEM, unless differently indicated. The differences in object exploration time were evaluated by a two-way analysis of variance (ANOVA). The differences in target-zone preference rate along the test epochs were assessed by two-way ANOVA for repeated measures (RM two-way ANOVA). The EOR index, difference score ON-BSL, anogenital sniffing and object exploration during the cued reward-conditioned learning, and total distance travelled were analysed by a two-tailed Student's $t$ test for unpaired measures, one-way ANOVA and two-way ANOVA, when appropriate. A Bonferroni post-hoc test was employed when necessary $(\alpha=0.05)$. Differences were considered statistically significant if $p<0.05$.

\section{Results}

\section{Experiment 1. EOR test: Validation}

Object exploration. We observed significant differences in the rats' exploration of the target object according to their pairing, or not, to the female encounter.

In particular, a two-way ANOVA on object exploration time, including reward-conditioning as the between-subject factor and object valence (novel versus familiar for the NoR group; emotional versus novel for the $\mathrm{R}$ group) as the within-subject factor, highlighted a significant effect of reward-conditioning $\left(F_{(1,36)}=\right.$ $12.00, p=0.0014)$, object valence $\left(F_{(1,36)}=52.86, p<0.001\right)$ and their interaction $\left(F_{(1,36)}=58.80 ; p<0.001\right)$. As expected, the NoR group spent significantly more time exploring the novel object then the target one $(t=10.56 ; p<0.001)$. On the other hand, the $\mathrm{R}$ group displayed a significant decrease in novel object-exploration time $(t=7.872 ; p<0.001)$ and a significant increase in emotionalobject exploration time $(t=2.972, p=0.0315)$ with respect to NoR group rats (Figure 2(a)). The acquisition of the reward-object association was also proven by the EOR index, expressed as normalisation to reference average value from NoR group rats: $R$ group rats showed a significantly higher EOR index with respect to the NoR group $(t=10.73 ; p<0.001 ; \mathrm{df}=18$ ) (Figure 2(b)).

The effects of female receptivity in the cued reward-conditioned learning and in the EOR test are reported in Table 1. When male rats were presented with a receptive female during the cued reward-conditioned learning, they showed a significant increase in socio-sexual behaviour, in terms of anogenital sniffing than when in the presence of a nonreceptive female rat $(p<0.001, t=$ $7.556, \mathrm{df}=18$, two-tailed Student's $t$ test). No differences in the exploration time of the target object were observed. When limbic memory was assessed in the EOR experiment, a two-way ANOVA showed significant effects of receptive female rat $\left(F_{(1,}\right.$ $\left.{ }_{36)}=13.31, p=0.0008\right)$, object valence $\left(F_{(1,36)}=6.288, p=\right.$ $0.0168)$ and their interaction $\left(F_{(1,36)}=8.931, p=0.005\right)$. In particular, rats exposed to the receptive female significantly increased the exploration of the emotional object $(t=4$.693, $p=$ 0.0002 , Bonferroni post-hoc test) and displayed a higher EOR index ( $p<0.001, t=6.7118, \mathrm{df}=18$, two-tailed Student's $t$ test) compared with male rats exposed to a nonreceptive female.

Target-zone preference. The nonpreferred zone of the Context A chamber was determined during epoch BSL for each animal, and the analysis of this behaviour showed neither effect of chamber's side nor significance among the animals. The nonpreferred zone was paired with the emotional (or target) object afterwards, and became the target zone. We recorded that significant differences in rats' preference for the target zone were dependent on the acquisition of the association between the rewarding experience with the receptive female rat and the target object.

In particular data analysis on the target-zone preference rate along the test epochs, with reward-conditioning as the betweensubject factor and the test epoch as the repeated-measure withinsubject factor, showed a significant effect of reward-conditioning $\left(F_{(1,18)}=17.00 ; p=0.0006\right)$, test epoch $\left(F_{(3,54)}=2.777, p=\right.$ $0.0499)$ and their interaction $\left(F_{(3,54)}=6.056 ; p=0.0012\right)$. Bonferroni post-hoc test revealed that NoR group rats did not significantly modify their zone preference after the presentation of the objects, and maintained a target-zone preference rate around zero along the test epochs. Notably, R group rats progressively increased their target-zone preference rate at the presentation of the objects, with a significant increase during epoch OFF and epoch ON-2 with respect to BSL $(t=3.280, p=0.0055 ; t=$ $4.236, p=0.0003$ ). When the effect of reward-conditioning was analysed, Bonferroni post-hoc test showed that $\mathrm{R}$ group rats displayed higher target-zone preference rate than NoR group rats during epoch OFF and ON-2 $(t=2.863, p=0.0220 ; t=5.370, p$ $<0.001$ ) (Figure 2(c)). A Student's $t$ test on the difference score ON-BSL also showed a higher score in the $\mathrm{R}$ group compared with NoR group rats $(t=7.564, p<0.001, \mathrm{df}=18)$ as a result of the longer time spent in the target zone in the presence of the emotional object (Figure 2(d)).

\section{Experiment 2. Effects of WIN on positive limbic memory in the EOR experiment}

Object exploration. We observed significant differences in rat approach behaviour to the emotional object as a consequence of the timing of WIN administration.

Drug administration at pre-conditioning time. When WIN was administered $30 \mathrm{~min}$ before the cued reward-conditioned learning, results of a two-way ANOVA on object exploration time, including treatment as the between-subject factor and object valence (emotional versus novel) as the within-subject factor, showed a significant effect of the interaction between treatment and object valence $\left(F_{(2,42)}=19.08, p<0.0001\right)$. The WIN group spent significantly longer time on the exploration of the emotional object than on the exploration of the novel one $(t=6.047, p<0.001)$. With reference to between-group differences, WIN rats displayed a significant decrease in the exploration of the novel object, when compared with the VEH group $(t$ $=2.563, p=0.0422$ ), this effect being prevented by AM281 pretreatment. AM281 + WIN rats showed no differences compared with VEH rats $(t=0.6030, p>0.999)$ and a significant increase in novel object exploration time, with respect to the WIN group $(t=3.166, p=0.0086)$. Moreover, the WIN group showed a significant increase in the exploration of the emotional object, with respect to VEH rats $(t=3.736, p=0.0017)$. Again, when rats were pre-treated with AM281, they showed no significant differences with respect to the VEH group $(t=1.491, p=0.4304)$ and a significant decrease in emotional-object exploration time when compared with the WIN group $(t=5.226, p<0.001$; Figure 3(a)). 


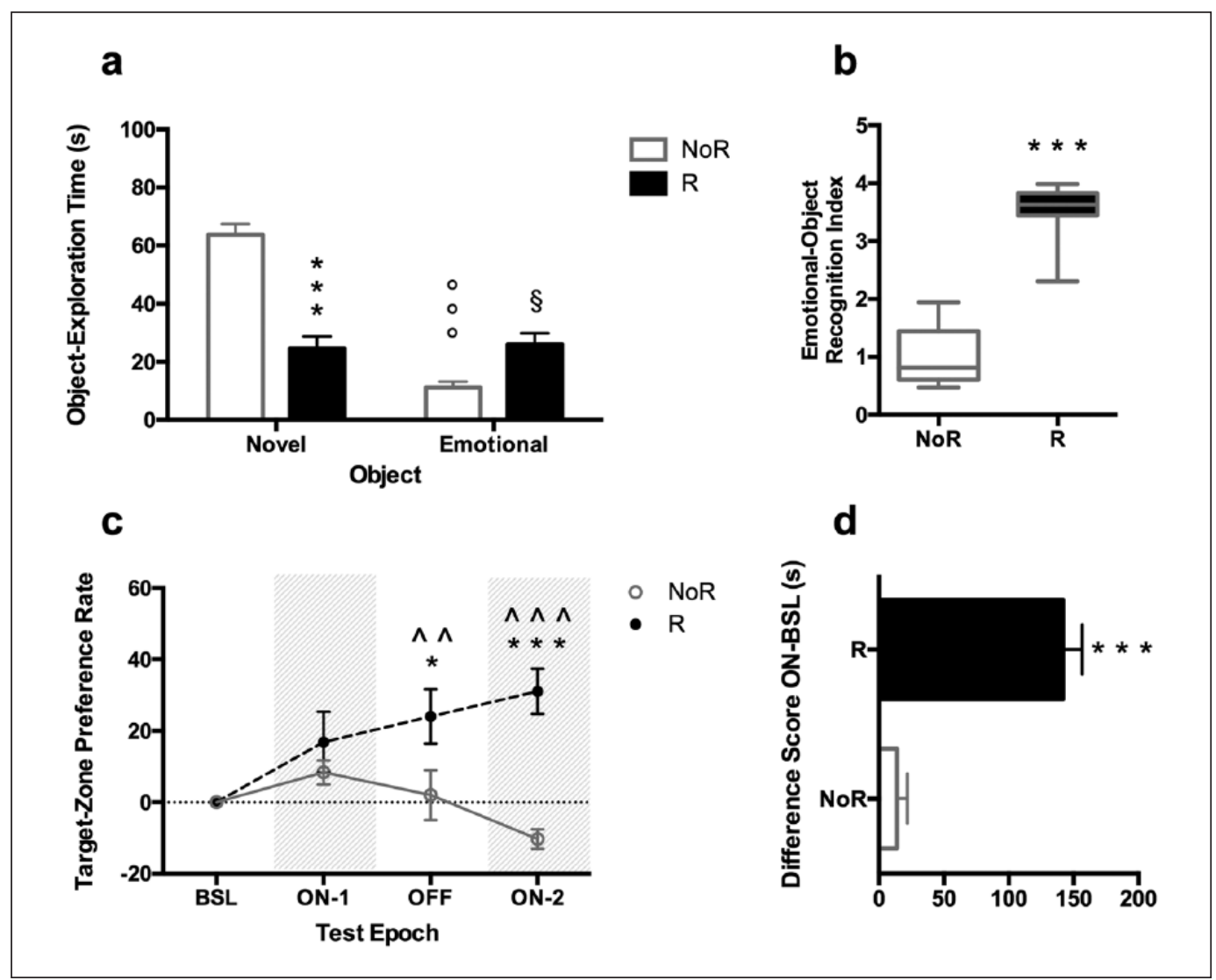

Figure 2. Emotional-object recognition test: Validation. Male rats were either exposed to the female encounter (reward; R group) or not (no reward; NoR group) during the cued reward-conditioned learning phase, and tested for positive limbic memory in the emotional-object recognition test. Effects of reward-conditioning on: (a) object exploration time; (b) emotional-object recognition index, expressed as normalisation to reference average value from NoR group rats; (c) target-zone preference rate, referring to the increase in the percentage of time spent in the target zone with respect to BSL; (d) difference score $0 \mathrm{~N}-\mathrm{BSL}$ (i.e. the difference between target-zone time during $0 \mathrm{~N}(\mathrm{ON}-1+0 \mathrm{~N}-2)$ and target-zone time during $\mathrm{BSL}$ ). Each value represents the mean \pm SEM of 10 rats. Each box-and-whisker plot represents the median (horizontal line in the box), $25-75 \%$ (box) and min-to-max (whiskers) values of 10 rats.

${ }^{*} p<0.05$.

${ }^{* * *} p<0.001$ versus respective NoR value;

${ }^{00} p<0.001$ versus novel NoR;

$\S_{p}<0.05$ versus emotional NoR;

$\hat{\wedge} p<0.05$;

${ }^{\wedge \wedge} p<0.001$ versus respective BSL.

BSL: test epoch baseline; NoR: no reward; R: reward; SEM: standard error of the mean.

Table 1. Effects of female receptivity in the cued reward-conditioned learning and in the emotional-object recognition experiment.

\begin{tabular}{lcr}
\hline Cued reward-conditioned learning & Nonreceptive female & Receptive female \\
\hline Socio-sexual behaviour & & $180.7 \pm 26.2^{* * *}$ \\
Anogenital sniffing (s) & $90.7 \pm 27.1$ & $133.7 \pm 19.3$ \\
Object exploratory behaviour & $135.0 \pm 27.2$ & \\
Object exploration (s) & & $26.0 \pm 12.1^{* * *}$ \\
Emotional-object recognition & $6.7 \pm 4.3$ & $24.5 \pm 13.1$ \\
Object exploration time & $22.7 \pm 1.6$ & $3.5 \pm 0.5^{* * *}$ \\
Emotional-object exploration (s) & $1.4 \pm 0.9$ &
\end{tabular}


Drug administration at post-conditioning time. When WIN was administered $30 \mathrm{~min}$ before the EOR experiment at post-conditioning time, a two-way ANOVA on object exploration time, including treatment as the between-subject factor and object valence (emotional versus novel) as the within-subject factor, indicated a significant effect of object valence $\left(F_{(1,42)}=8.130, p=\right.$ $0.0067)$ and the interaction between treatment and object valence $\left(F_{(2,42)}=8.604, p=0.0007\right)$. Post-conditioning WIN rats showed a significant decrease in the exploration of the emotional object, with respect to the novel one $(t=4.803, p<0.0001)$. Moreover, post-conditioning WIN rats increased novel object exploration time with respect to VEH rats $(t=4.170, p=0.0004)$; this effect was not observed when rats were pre-treated with AM281. In fact, post-conditioning AM281 + WIN rats showed no differences with respect to VEH rats $(t=1.176, p=0.7388)$ and a significant decrease in novel object exploration time, when compared with WIN group $(t=2.994, p=0.0138$; Figure $3(\mathrm{~b})$ ).

Effect of drug administration time. A two-way ANOVA on object exploration time following pre- and post-conditioning WIN administration, including administration time as the between-subject factor and object valence (emotional versus novel) as the withinsubject factor, showed a significant effect of the interaction between administration time and object valence $\left(F_{(1,28)}=44.19, p<0.001\right)$. In particular, post-conditioning WIN administration decreased the exploration time of the emotional object $(t=4.068, p=0.0021)$ and increased the exploration time of the novel object $(t=5.333, p<$ $0.0001)$ with respect to pre-conditioning WIN administration.

When the effect of pre- and post-conditioning WIN was assessed on the EOR index, a two-way ANOVA with treatment as the between-subject factor and administration time as the withinsubject factor, indicated a significant effect of administration time $\left(F_{(1,42)}=8.254, p=0.0064\right)$ and the interaction between treatment and administration time $\left(F_{(2,42)}=11.32, p=0.0001\right)$. In particular, pre-conditioning WIN administration significantly increased the EOR index when compared with pre-conditioning $\operatorname{VEH}(t=2.965, p=0.0149)$; whereas pre-conditioning AM281 + WIN rats showed no differences with respect to VEH rats $(t=$ $0.9197, p>0.999)$ and a decreased EOR index with respect to the WIN group $(t=3.884, p=0.0011)$. On the other hand, post-conditioning WIN rats showed a significantly decreased EOR index with respect to post-conditioning $\mathrm{VEH}$ rats $(t=3.057, p=$ 0.0116). Moreover, while no differences were observed between pre- and post-conditioning VEH administration $(t=0.4860, p>$ $0.999)$, and between pre- and post-conditioning AM $281+\mathrm{WIN}(t$ $=0.07385, p>0.999)$ treatment, post-conditioning WIN rats displayed a decreased EOR index $(t=5.536, p<0.0001)$ when compared with the pre-conditioning WIN group ( Figure 3(e)).

Target-zone preference. According to the previous experiment, we observed that significant differences in rat preference for the target zone were dependent on the timing of WIN administration.

Drug administration at pre-conditioning time. When WIN was administered $30 \mathrm{~min}$ before the cued reward-conditioned learning, the effect of the pre-conditioning treatment on target-zone preference rate, was analysed by a RM two-way ANOVA, including pre-conditioning treatment as the between-subject factor and test epoch as the repeated-measure within-subject factor. The analysis showed a significant effect of treatment $\left(F_{(2,21)}=6.281 ; p=0.0073\right)$ and test epoch $\left(F_{(3,63)}=24.08 ; p<0.0001\right)$. Notably, at the presentation of the emotional object, rats progressively increased their target-zone preference along the test epochs. In particular, VEH rats increased their target-zone preference rate on epochs ON-1 $(t=$ $2.556, p=0.0490)$, OFF $(t=3.168, p=0.0071)$ and ON-2 $(t=3.834$, $p=0.0009)$ with respect to BSL values; WIN rats displayed an even higher target-zone preference rate on epochs ON-1 $(t=5.108, p$ $<0.0001)$, OFF $(t=5.842, p<0.0001)$ and ON-2 $(t=5.729, p<$ $0.0001)$ with respect to BLS values, and significantly higher values than VEH on epoch ON-1 $(t=2.497, p=0.0434)$ and OFF $(t=2.505$, $p=0.0425)$. The WIN effect on target-zone preference rate was actually prevented by AM281 pre-treatment, that decreased target-zone preference rate on epochs ON-1 $(t=3.108, p=0.0077)$ and ON-2 $(t=2.474, p=0.0461)$ with respect to WIN values ( Figure 3(c)).

Drug administration at post-conditioning time. When WIN was administered 30 min before the EOR experiment (postconditioning), a RM two-way ANOVA on target-zone preference rate, considering post-conditioning treatment as the between-subject factor and test epoch as the repeated-measure within-subject factor, highlighted a significant effect of treatment $\left(F_{(2,21)}=10.27\right.$; $p=0.0008)$, test epoch $\left(F_{(3,63)}=11.35, p<0.001\right)$ and their interaction $\left(F_{(6,63)}=2.426, p=0.0358\right)$. VEH rats increased their targetzone preference rate during $\mathrm{ON}-1$, OFF and $\mathrm{ON}-2$ with respect to BSL $(t=2.889, p=0.0159 ; t=4.987, p<0.001 ; t=3.437$, $p=0.0031)$ whereas WIN rats did not modify their target-zone preference rate at the presentation of the objects and showed lower values on epoch OFF $(t=3.665, p=0.0013)$ and ON-2 $(t=3.613$, $p=0.0015)$ compared with VEH. The WIN effect on target-zone preference rate was prevented by AM281 pre-treatment, which increased target-zone preference rate during epoch $\mathrm{ON}-1(t=$ 2.553, $p=0.0375)$ and ON-2 $(t=3.879, p=0.0006)$ (Figure 3(d)).

\section{Effect of drug administration time}

A RM two-way ANOVA on target-zone preference rate compared data from pre- and post-conditioning WIN-administered rats. WIN administration time was the between-subject factor and test epoch was the repeated-measure within-subject factor. The analysis showed a significant effect of WIN administration time $\left(F_{(1,14)}=\right.$ $24.06, p=0.0002)$, test epoch $\left(F_{(3,42)}=5.986, p=0.0017\right)$ and their interaction $\left(F_{(3,42)}=3.918, p=0.0149\right)$ : post-conditioning WIN decreased target-zone preference rate during epochs ON-1 $(t=$ 2.913, $p=0.0205)$, OFF $(t=3.391, p=0.0064)$ and ON-2 $(t=$ $4.506, p=0.001)$, when compared with pre-conditioning WIN.

When the effect of pre- and post-conditioning WIN was assessed on the difference score ON-BSL, two-way ANOVA considering treatment as the between-subject factor and administration time as the within-subject factor, revealed a significant effect of administration time $\left(F_{(1,42)}=4.588, p=0.0381\right)$ and interaction between treatment and administration time $\left(F_{(2,42)}=9.452, p=\right.$ 0.0004). In particular, Bonferroni post-hoc test highlighted an increase in difference score ON-BSL in pre-conditioning WIN group when compared with post-conditioning WIN rats $(t=4.571$, $p=0.0006)$. Moreover, a significant decrease in the difference score ON-BSL in post-conditioning WIN rats was observed with respect to post-conditioning $\mathrm{VEH}(t=3.210, p=0.0381)$ and postconditioning AM281 + WIN group $(t=4.034, p=0.0034)$. AM218 + WIN rats did not display significant differences in difference score ON-BSL with respect to VEH rats ( Figure 3(f)). 


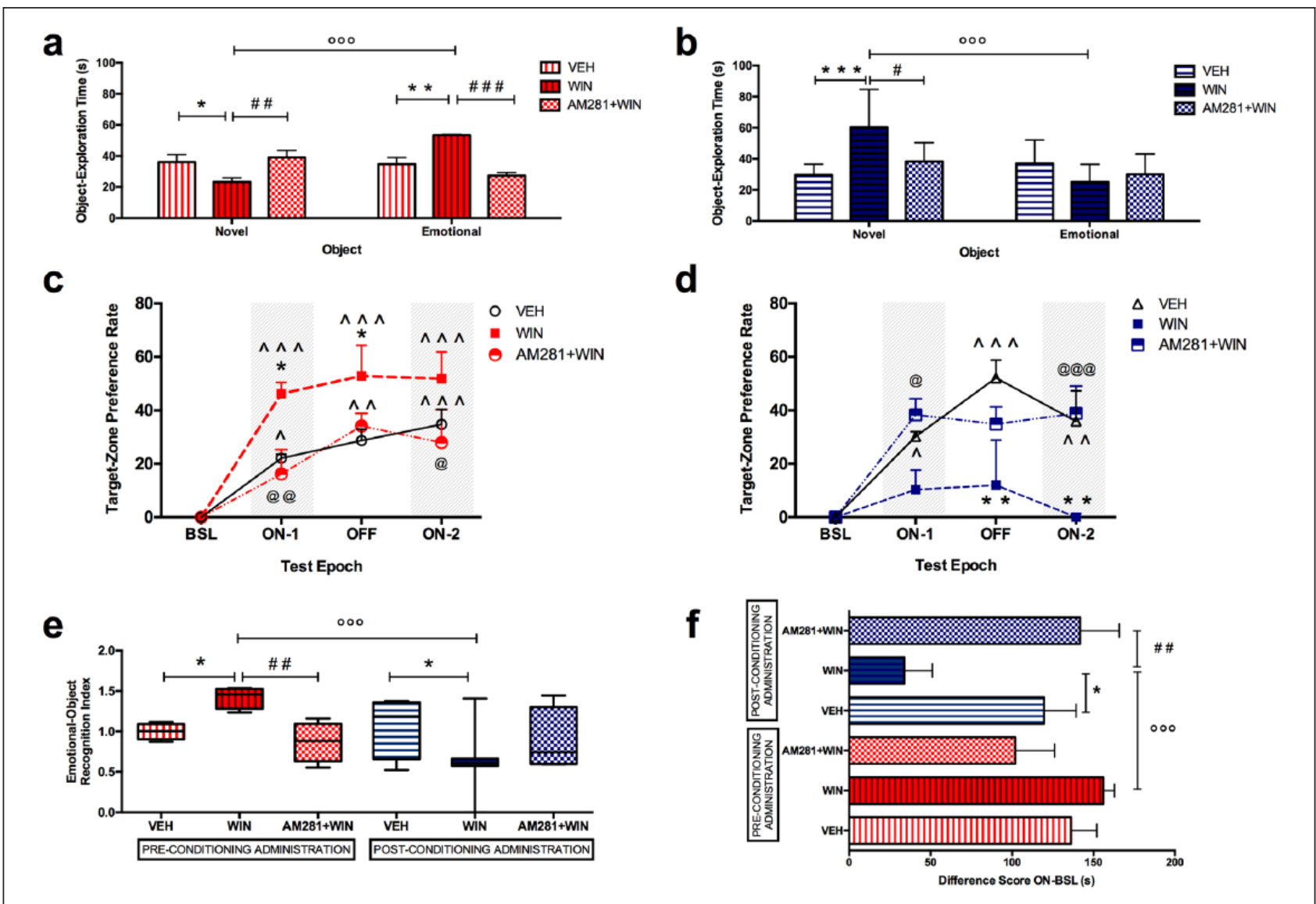

Figure 3. Effects of WIN in the emotional-object recognition test. (a) Effects of pre-conditioning WIN administration on object exploration time; (b) effects of post-conditioning WIN administration on object exploration time; (c) effects of pre-conditioning WIN administration on target-zone preference rate; $(d)$ effects of post-conditioning WIN administration on target-zone preference rate; (e) effects of pre- and post-conditioning WIN administration on emotional-object recognition index, expressed as normalisation to reference average value from pre-conditioning VEH rats; $(f)$ effects of pre- and post-conditioning WIN administration on difference score ON-BSL (i.e. the difference between target-zone time during ON (ON-1 $+0 \mathrm{~N}-2$ ) and target-zone time during BSL). Each bar represents the mean \pm SEM of eight rats. Each box-and-whisker plot represents the median (horizontal line in the box), 25-75\% (box) and min-to-max (whiskers) values of eight rats.

${ }^{*} p<0.05$;

${ }^{* *} p<0.01$;

${ }^{* * *} p<0.001$ versus respective VEH;

${ }^{\circ} p<0.001 ;$

$\# p<0.05$;

$\# p<0.01 ;$

$\# \#$ \# 0.001

$\wedge p<0.05$;

${ }^{\wedge \wedge} p<0.01$;

${ }^{\wedge \wedge} p<0.001$ versus BSL;

@ $p<0.05$;

$@ p<0.01$ versus WIN.

BSL: test epoch baseline; VEH: vehicle; WIN: WIN 55,212-2.

\section{Socio-sexual and object exploratory behaviour}

When WIN was administered at pre-conditioning time, no significant effects on male rats' behaviour were recorded. In particular, the analysis of data from anogenital sniffing of the female rat and object exploration during the cued reward-conditioned learning showed no significant differences between the WIN and VEH groups (Table 2).

\section{Experiment 3. Behavioural reactivity assessment}

In order to assess whether the emotional experience altered male rats' behavioural reactivity, locomotor activity during epoch BSL in the EOR experiment was measured. A one-way ANOVA indicated no significant effect of female receptivity on total distance travelled among the three experimental groups $\left(F_{(2,27)}=0.1573, p=0.8553\right)$. 
Table 2. Effects of WIN on socio-sexual and object exploratory behaviour during the cued reward-conditioned learning.

\begin{tabular}{|c|c|c|c|c|}
\hline \multirow[t]{2}{*}{ Measures } & VEH & WIN & \multirow[t]{2}{*}{$p$-value } & \multirow[t]{2}{*}{$t ; \mathrm{df}$} \\
\hline & \multicolumn{2}{|l|}{ Mean \pm SEM } & & \\
\hline \multicolumn{5}{|l|}{ Socio-sexual behaviour } \\
\hline Anogenital sniffing (s) & $170.7 \pm 14.9$ & $199.0 \pm 5.6$ & ns & $t=1.78 ; \mathrm{df}=14$ \\
\hline \multicolumn{5}{|c|}{ Object exploratory behaviour } \\
\hline Object exploration (s) & $119.5 \pm 20.8$ & $144.3 \pm 6.1$ & ns & $t=1.14 ; \mathrm{df}=14$ \\
\hline
\end{tabular}

df: degrees of freedom; ns: not significant; SEM: standard error of the mean; VEH: vehicle; WIN: WIN 55,212-2.

Moreover, in order to control for potential WIN-induced motor impairment that might affect rat reactivity during the EOR test, locomotor activity of pre- and post-WIN and VEH rats at BSL was measured. A two-way ANOVA on total distance travelled showed no significant effect of WIN treatment $\left(F_{(1,28)}=\right.$ $0.038, p=0.8468)$, administration time $\left(F_{(1,28)}=0.02408, p=\right.$ $0.6275)$ or their interaction $\left(F_{(1,28)}=1.25, p=0.2731\right)$.

\section{Discussion}

The current study explored the acquisition of positive limbic memory in male rats, in a cued reward-conditioned learning task, and the effect of the activation of cannabinoid signalling, by the administration of WIN, a cannabinoid receptor agonist.

In the present experimental conditions, the reward-conditioning consisted in exposing male rats to an encounter with a receptive female rat in the presence of a target object. The association with the positive experience turned the target object into an 'emotional object' because of the formation of positive limbic memory (Ramirez et al., 2015).

The recognition of the emotional object as a 'rewarding memory' was measured by the animal's approach towards it, in the face of the presence of a novel object. Indeed, novelty is attractive, may actually represent a form of intrinsic reward and also interacts with reward motivation inducing approach behaviour (Corr, 2004; Krebs et al., 2009). When in the presence of both a familiar but emotionally salient object and a totally novel one, the $\mathrm{R}$ group spent the same amount of time on the exploration of each object. This result suggests that the acquisition and retrieval of the reward-object association subtracted value to novelty exploration, increasing the approach behaviour to the familiar emotional object, and witnesses the functioning of rat discriminative processes that follow the formation of reward limbic memory. It is not the scope of this research in establishing a graduation of salience between natural reinforcements (novelty versus socio-sexual-related memory), rather highlighting the higher motivational valence of the emotional object observed in the $\mathrm{R}$ group with respect to the familiar valence of the NoR target object displayed by NoR group rats. Indeed, as expected, when the object was not paired with the female rat, and then was not acquired as an emotional object, its incentive value was lower than a novel object and rats were driven by novelty-seeking and strongly preferred the novel object. Furthermore, when male rats were exposed to a nonreceptive female rat, socio-sexual behaviour decreased, as well as emotional object exploration and recognition, pointing at female receptivity as the critical factor in the acquisition of the positive value of the emotional object.

The EOR experiment was carried out in a separate setting than the cued reward-conditioned learning in order to distinguish the valence of the emotional object from the valence of the location. Since we employed a single-trial conditioning procedure, we took advantage of a biased stimulus-place association (Prus et al., 2009) in order to maximize the potential shift in place preference and unveil subtle changes in animal behavioural response (Bozarth, 1987). In fact, once the animals exhibited their individual innate preference for one side of the Context A chamber, the nonpreferred zone, called the target zone, hosted the emotional object. Notably, the positioning of the emotional object inverted the preference for the target zone of the Context A chamber; from being the least preferred during epoch BSL, it became the most visited afterwards.

This result suggests that animals responded actively to the manipulations of the environment and that motivational factors positively influence the integration and discrimination of intrinsic emotional inputs and explicit cognitive cues in the EOR test. Actually, the animals exploited their ability of memorising and discriminating the objects on the basis of their visual sensory competence, which was integrated with emotional elaboration and attribution of incentive salience. The employment of the EOR test enhances the investigation of emotional memory, and integrates the information obtained by conditioned place preference/aversion experiments, where reward or fear-related responses might be blunted by drug effects or relevant environmental cues (Cunningham and Zerizef, 2014).

When it comes to defining the neurobiological basis of memory formation, it is increasingly clear that the framework is complex and depends on several factors, such as the nature of the task (emotional or non-emotional), the memory stage investigated (acquisition, retrieval and extinction), and the experimental model. Among the main neurochemical modulators involved, cannabinoids exert relevant effects on memory and the underlying neural plasticity, with reports of both memory impairments on non-emotional memory (such as spatial learning) and memory facilitation when an emotional valence is involved (such as extinction learning) (Akirav, 2011; Chhatwal and Ressler, 2007).

Therefore, in this study, we aimed to investigate the consequences of the variation in the relationship among the internal emotional milieu, rewarding positive experience and explicit memory by the stimulation of cannabinoid signalling.

Our present results show that, when administered systemically at pre-conditioning time, WIN 55,212-2, a widely used nonselective cannabinoid agonist (Citraro et al., 2016; Florek-Luszczki et al., 2014), employed at a dose that does not impair behavioural reactivity (Brancato et al., 2016; Darmani, 2001; Järbe, 2006), induced a significant enhancement in the approach behaviour to the emotional object in the face of a reduction in the exploration of the novel object. Moreover, pre-conditioning WIN-treated rats 
increased their preference for the target zone when it hosted the emotional object at a higher rate than VEH rats. This evidence suggests that the activation of cannabinoid signalling facilitated the formation of memory traces of the emotional object, likely by enhancing the value of the association between the rewarding stimulus and the object and prioritising this information above the incentive value of novelty. The WIN effect seems to be mediated by its activity on $\mathrm{CB} 1$ receptors, since the administration of the CB1 antagonist AM281 was able to counteract WIN impact on rewarding limbic memory.

Interestingly, when WIN was administered at post-conditioning time, rats spent a shorter time in the exploration of the emotional object with respect to the novel one, suggesting that post-conditioning cannabinoid stimulation might dampen the retrieval of the association between the positive emotional experience and the object. Again, the pre-treatment with CB1 antagonist AM281, prior to post-conditioning WIN administration, was able to counteract the WIN effect on object recognition, further pointing to $\mathrm{CB} 1$ signalling as the pathway involved in the modulation of reward-related limbic memory.

However, the idea that the post-conditioning timing of cannabinoid system stimulation, rather than impairing retrieval of retrograde memory traces, might determine an upgrade in positive value attribution for a subsequent motivational experience (i.e. novelty exploration), cannot be ruled out. Thus, it is intriguing to speculate that $\mathrm{CB} 1$ receptor activation prior to the exposure to a positive natural stimulus, is able to potentiate its incentive motivational connotation whatever its original value. Further studies on the neurobiological background involving diverse manipulation of the endocannabinoid system will clarify the cellular and molecular features that underlie this behavioural evidence.

The WIN-induced effect on limbic memory formation seems to depend on the valence of the emotional experience. Indeed, this group of research has recently reported that pre-conditioning WIN administration induces a decline in encoding and retrieval of limbic memory traces when the emotional experience is aversive (Brancato et al., 2016). Altogether, these data point to cannabinoid activation as a condition that reinforces the encoding and retrieval of subsequent positive emotional experiences, prioritises information in memory according to value attribution and decreases the formation of aversive limbic memory. It is not our ambition to give an epistemological interpretation of these results but perhaps, the high incidence of cannabis use in the population, especially in adolescence, is not only due to its euphoric, talkative, loosening effects (Schierenbeck et al., 2008), but also to the potential increase in value attribution of positive experiences and their storing in memory, and at the same time to the impairment in the formation of unpleasant aversive memory traces (Lutz et al., 2015; Parsons and Hurd, 2015).

Lastly, the possibility to assess discrete selective effects of substances on different forms of learning and memory in the animal model by the combined employment of specific memory tasks, represents a strategy that increases the translational value of the preclinical research in behavioural science, and can help in unveiling the addictive and confounding properties of many psychotropic drugs.

\section{Declaration of conflicting interest}

The author(s) declared no potential conflicts of interest with respect to the research, authorship, and/or publication of this article.

\section{Funding}

The author(s) disclosed receipt of the following financial support for the research, authorship, and/or publication of this article: This research was supported by a 2012-ATE-0167 grant from the University of Palermo, Italy.

\section{References}

Ågmo A and Berenfeld R (1990) Reinforcing properties of ejaculation in the male rat: Role of opioids and dopamine. Behav Neurosci 104: $177-182$.

Akirav I (2011) The role of cannabinoids in modulating emotional and non-emotional memory processes in the hippocampus. Front Behav Neurosci 5: 34.

Asensio S, Romero MJ, Palau C, et al. (2010) Altered neural response of the appetitive emotional system in cocaine addiction: An fMRI Study. Addict Biol 15: 504-516.

Bardo MT and Bevins RA (2000) Conditioned place preference: What does it add to our preclinical understanding of drug reward? Psychopharmacology 153: 31-43.

Bozarth MA (1987) Neuroanatomical boundaries of the reward-relevant opiate-receptor field in the ventral tegmental area as mapped by the conditioned place preference method in rats. Brain Res 414: 77-84.

Brancato A, Plescia F, Marino RA, et al. (2014) Involvement of dopamine D2 receptors in addictive-like behaviour for acetaldehyde. PLoS One 9(6): e99454.

Brancato A, Lavanco G, Cavallaro A, et al. (2016a) The use of the emotional-object recognition as an assay to assess learning and memory associated to an aversive stimulus in rodents. J Neurosci Methods 274: 106-115.

Brancato A, Plescia F, Lavanco G, et al. (2016b) Continuous and Intermittent Alcohol Free-Choice from Pre-gestational Time to Lactation: Focus on Drinking Trajectories and Maternal Behavior. Front Behav Neurosci 10: 31

Brown MW, Warburton EC and Aggleton JP (2010) Recognition memory: Material, processes, and substrates. Hippocampus 20: 1228-1244.

Byers SL, Wiles MV, Dunn SL, et al. (2012) Mouse estrous cycle identification tool and images. PLoS One 7: e35538.

Cacace S, Plescia F, La Barbera M, et al. (2011) Evaluation of chronic alcohol self-administration by a 3-bottle choice paradigm in adult male rats. Effects on behavioural reactivity, spatial learning and reference memory. Behav Brain Res 219: 213-220.

Cacace S, Plescia F, Barberi I, et al. (2012) Acetaldehyde oral selfadministration: Evidence from the operant-conflict paradigm. Alcohol Clin Exp Res 36: 1278-1287.

Cannizzaro C, Plescia F, Gagliano M, et al. (2007) Effects of pre- and postnatal exposure to 5-methoxytryptamine and early handling on an object-place association learning task in adolescent rat offspring. Neurosci Res 59: 74-80.

Cannizzaro C, La Barbera M, Plescia F, et al. (2010) Ethanol modulates corticotropin releasing hormone release from the rat hypothalamus: Does acetaldehyde play a role? Alcohol Clin Exp Res 34: 588-593.

Cannizzaro C, Malta G, Argo A, et al. (2016) Behavioural and pharmacological characterization of a novel cannabinomimetic adamantanederived indole, APICA, and considerations on the possible misuse as a psychotropic spice abuse, in $\mathrm{C} 57 \mathrm{~b} 1 / 6 \mathrm{~J}$ mice. Forensic Sci Int 265: 6-12.

Cannizzaro E, Martire M, Gagliano M, et al. (2005) Reversal of prenatal diazepam-induced deficit in a spatial-object learning task by brief, periodic maternal separation in adult rats. Behav Brain Res 161:320-330.

Chao OY, Nikolaus S, Lira Brandão $M$, et al. (2017) Interaction between the medial prefrontal cortex and hippocampal CA1 area is essential for episodic-like memory in rats. Neurobiol Learn Mem 141: 72-77.

Chhatwal JP and Ressler KJ (2007) Modulation of fear and anxiety by the endogenous cannabinoid system. CNS Spectr 12: 211-220. 
Christianson SA and Engelberg E (1997) Complex memory function for mental trauma. Memory and forgetting ae necessary for the emotional adaptation process. Lakartidningen 94: 1721-1724.

Chu X and Ågmo A (2015) Sociosexual behaviors during the transition from non-receptivity to receptivity in rats housed in a seminatural environment. Behav Processes 113: 24-34.

Citraro R, Russo E, Leo A, et al. (2016) Pharmacokinetic-pharmacodynamic influence of N-palmitoylethanolamine, arachidonyl-2'-chloroethylamide and WIN 55,212-2 on the anticonvulsant activity of antiepileptic drugs against audiogenic seizures in DBA/2 mice. Eur $J$ Pharmacol 791: 523-534.

Cohen MX and Ranganath C (2005) Behavioral and neural predictors of upcoming decisions. Cogn Affect Behav Neurosci 5: 117-126.

Corr PJ (2004) Reinforcement sensitivity theory and personality. Neurosci Biobehav Rev 28: 317-332.

Cunningham CL and Zerizef CL (2014) Effects of combining tactile with visual and spatial cues in conditioned place preference. Pharmacol Biochem Behav 124: 443-450.

Darmani NA (2001) The cannabinoid CB1 receptor antagonist SR $141716 \mathrm{~A}$ reverses the antiemetic and motor depressant actions of WIN 55,212-2. Eur J Pharmacol 430: 49-58.

De Carvalho CR, Pamplona FA, Cruz JS, et al. (2014) Endocannabinoids underlie reconsolidation of hedonic memories in Wistar rats. Psychopharmacology 231: 1417-1425.

Fang Q, Li FQ, Li YQ, et al. (2011) Cannabinoid CB1 receptor antagonist rimonabant disrupts nicotine reward-associated memory in rats. Pharmacol Biochem Behav 99: 738-742.

Florek-Luszczki M, Wlaz A, Kondrat-Wrobel MW, et al. (2014) Effects of WIN 55,212-2 (a non-selective cannabinoid CB1 and CB 2 receptor agonist) on the protective action of various classical antiepileptic drugs in the mouse $6 \mathrm{~Hz}$ psychomotor seizure model. $J$ Neural Transm 121: 707-715.

Forbes NF, Stewart CA, Matthews K, et al. (1996) Chronic mild stress and sucrose consumption: Validity as a model of depression. Physiol Behav 60: 1481-1484.

Hahn T, Dresler T, Ehlis AC, et al. (2009) Neural response to reward anticipation is modulated by Gray's impulsivity. Neuroimage 46: 1148-1153.

Harb MR, Sousa N, Zihl J, et al. (2014) Reward components of feeding behaviour are preserved during mouse aging. Front Aging Neurosci 6: 242 .

Harding SM and McGinnis MY (2004) Androgen receptor blockade in the MPOA or VMN: Effects on male sociosexual behaviors. Physiol Behav 81: 671-680.

Hughes AM, Everitt BJ and Herbert J (1990) Comparative effects of preoptic area infusions of opioid peptides, lesions and castration on sexual behaviour in male rats: Studies of instrumental behaviour, conditioned place preference and partner preference. Psychopharmacology 102: 243-256.

Järbe TU, Ross T, DiPatrizio NV, et al. (2006) Effects of the CB1R agonist WIN-55,212-2 and the CB1R antagonists SR-141716 and AM-1387: Open-field examination in rats. Pharmacol Biochem Behav 85: 243-252.

Karimi S, Azizi P, Shamsizadeh A, et al. (2013) Role of intra-accumbal cannabinoid CB1 receptors in the potentiation, acquisition and expression of morphine-induced conditioned place preference. Behav Brain Res 247: 125-131.

Kelley BJ, Yeager KR, Pepper TH, et al. (2005) Cognitive impairment in acute cocaine withdrawal. Cogn Behav Neurol 18: 108-112.

Kippin TE and van der Kooy D (2003) Excitotoxic lesions of the tegmental pedunculopontine nucleus impair copulation in naive male rats and block the rewarding effects of copulation in experienced male rats. Eur J Neurosci 18: 2581-2591.

Krebs R, Schott BH and Düzel E (2009) Personality traits are differentially associated with patterns of reward and novelty processing in the human substantia nigra/ventral tegmental area. Biol Psychiatry 65: 103-110.
Lang PJ, Davis M and Ohman A (2000) Fear and anxiety: Animal models and human cognitive psychophysiology. J Affect Disord 61: $137-159$.

LeDoux JE (1992) Brain mechanisms of emotion and emotional learning. Curr Opin Neurobiol 2: 191-197.

LeDoux JE (2000) Emotion circuits in the brain. Annu Rev Neurosci 23: 155-184.

Loureiro M, Renard J, Zunder J, et al. (2015) Hippocampal cannabinoid transmission modulates dopamine neuron activity: Impact on rewarding memory formation and social interaction. Neuropsychopharmacology 40: 1436-1447.

Loureiro M, Kramar C, Renard J, et al. (2016) Cannabinoid transmission in the hippocampus activates nucleus accumbens neurons and modulates reward and aversion-related emotional salience. Biol Psychiatry 80: 216-225.

Lutz B, Marsicano G, Maldonado R, et al. (2015) The endocannabinoid system in guarding against fear, anxiety and stress. Nat Rev Neurosci 16: 705-718.

Marsicano G, Wotjak CT, Azad SC, et al. (2002) The endogenous cannabinoid system controls extinction of aversive memories. Nature 418: 530-534.

Mehrara BJ and Baum MJ (1990) Naloxone disrupts the expression but not the acquisition by male rats of a conditioned place preference response for an oestrous female. Psychopharmacology 101: $118-125$.

Miller RL and Baum MJ (1987) Naloxone inhibits mating and conditioned place preference for an estrous female in male rats soon after castration. Pharmacol Biochem Behav 26: 781-789.

Murphy A, Taylor E and Elliott R (2012) The detrimental effects of emotional process dysregulation on decision-making in substance dependence. Front Integr Neurosci 6: 101.

Niendam TA, Laird AR, Ray KL, et al. (2012) Meta-analytic evidence for a superordinate cognitive control network subserving diverse executive functions. Cogn Affect Behav Neurosci 12: 241-268.

Ochsner KN and Gross JJ (2005) The cognitive control of emotion. Trends Cogn Sci 9: 242-249.

Paredes RG (2009) Evaluating the neurobiology of sexual reward. ILAR $J$ 50: $15-27$.

Parsons LH and Hurd YL (2015) Endocannabinoid signalling in reward and addiction. Nat Rev Neurosci 16: 579-594.

Peretto P and Paredes RG (2014) Social cues, adult neurogenesis, and reproductive behavior. In: Mucignat-Caretta C (ed.) Neurobiology of Chemical Communication. Boca Raton, FL: CRC Press, Taylor \& Francis.

Plescia F, Brancato A, Marino RA, et al. (2013) Acetaldehyde as a drug of abuse: Insight into AM281 administration on operant-conflict paradigm in rats. Front Behav Neurosci 7: 64.

Plescia F, Brancato A, Marino RA, et al. (2014a) Effect of acetaldehyde intoxication and withdrawal on NPY expression: Focus on endocannabinoidergic system involvement. Front Psychiatry 5: 138.

Plescia F, Marino RA, Navarra M, et al. (2014b) Early handling effect on female rat spatial and non-spatial learning and memory. Behav Processes 103: 9-16.

Plescia F, Brancato A, Venniro M, et al. (2015) Acetaldehyde selfadministration by a two-bottle choice paradigm: Consequences on emotional reactivity, spatial learning, and memory. Alcohol 49: 139-148.

Prus AJ, James JR and Rosecrans JA (2009) Chapter 4: Conditioned place preference. In: Buccafusco JJ (ed.) Methods of Behavior Analysis in Neuroscience. 2nd ed. Boca Raton, FL: CRC Press, Taylor $\&$ Francis.

Ramirez S, Liu X, MacDonald CJ, et al. (2015) Activating positive memory engrams suppresses depression-like behaviour. Nature 522: 335-339.

Rizzo V, Carletti F, Gambino G, et al. (2014) Role of CB2 receptors and cGMP pathway on the cannabinoid-dependent antiepileptic 
effects in an in vivo model of partial epilepsy. Epilepsy Res 108: 1711-1718.

Schierenbeck T, Riemann D, Berger M, et al. (2008) Effect of illicit recreational drugs upon sleep: Cocaine, ecstasy and marijuana. Sleep Med Rev 12: 381-389.

Shin LM and Liberzon I (2010) The neurocircuitry of fear, stress, and anxiety disorders. Neuropsychopharmacology 35: 169-191.

Suenaga T and Ichitani Y (2008) Effects of hippocampal administration of a cannabinoid receptor agonist WIN 55,212-2 on spontaneous object and place recognition in rats. Behav Brain Res 190: $248-252$.

Tonegawa S, Pignatelli M, Roy DS, et al. (2015) Memory engram storage and retrieval. Curr Opin Neurobiol 35: 101-119.

Trezza V, Campolongo P and Vanderschuren LJ (2011) Evaluating the rewarding nature of social interactions in laboratory animals. Dev Cogn Neurosci 1: 444-458.

Tzschentke TM (1998) Measuring reward with the conditioned place preference paradigm: A comprehensive review of drug effects, recent progress and new issues. Prog Neurobiol 56: 613-672.
Tzschentke TM (2007) Measuring reward with the conditioned place preference (CPP) paradigm: Update of the last decade. Addict Biol 12: $227-462$.

Volkow ND, Wang GJ, Fowler JS, et al. (2012) Addiction circuitry in the human brain. Annu Rev Pharmacol Toxicol 52: 321-336.

Wang JJ, Yao WQ, Chen YJ, et al. (2014) Neurons in NAc core and BLA are activated during cocaine context-associated reward memory retrieval in mice. Sheng Li Xue Bao 66: 545-558.

Wells AM, Lasseter HC, Xie X, et al. (2011) Interaction between the basolateral amygdala and dorsal hippocampus is critical for cocaine memory reconsolidation and subsequent drug context-induced cocaine-seeking behavior in rats. Learn Mem 18: 693-702.

Yu LL, Wang XY, Zhao M, et al. (2009) Effects of cannabinoid CB1 receptor antagonist rimonabant in consolidation and reconsolidation of methamphetamine reward memory in mice. Psychopharmacology 204: 203-211.

Zhang X, Cui Y, Jing J, et al. (2011) Involvement of p38/NF- $\mathrm{kB}$ signaling pathway in the nucleus accumbens in the rewarding effects of morphine in rats. Behav Brain Res 218: 184-189. 